\title{
Variations
}

Variations

Revue internationale de théorie critique

16 | 2012

Tahrir is here !

\section{Égypte : grèves de masse, espace public oppositionnel et insurrection des consciences}

Jacques Chastaing

\section{(2) OpenEdition}

\section{Journals}

Édition électronique

URL : http://journals.openedition.org/variations/192

DOI : 10.4000/variations. 192

ISSN : 1968-3960

Éditeur

Les amis de Variations

Édition imprimée

Date de publication : 16 janvier 2012

\section{Référence électronique}

Jacques Chastaing, «Égypte: grèves de masse, espace public oppositionnel et insurrection des

consciences », Variations [En ligne], 16 | 2012, mis en ligne le 20 février 2012, consulté le 19 avril 2019. URL : http://journals.openedition.org/variations/192 ; DOI : 10.4000/variations.192

Ce document a été généré automatiquement le 19 avril 2019.

Les ami•e•s de Variations 


\section{Égypte : grèves de masse, espace public oppositionnel et insurrection des consciences}

Jacques Chastaing

\section{NOTE DE L'ÉDITEUR}

Première publication sur www.theoriecritique.com, « Tahrir is here ! », 16 janvier 2012, pp. 25-48

Le 10 décembre 2011.

\section{Une révolution qui continue et s'approfondit, révélatrice de transformations mondiales}

1 La révolution égyptienne associe dans un même temps un espace oppositionnel public très largement visible, place Tahrir et internet, un peu moins avec les journaux muraux urbains, une insurrection des consciences qui révise les hiérarchies des valeurs personnelles et bouscule les relations matrimoniales au sein de la famille, et, enfin, des vagues de manifestations et grèves massives, parfois économiques, fréquemment politiques, le plus souvent les deux à la fois.

2 Le plus frappant, même si cette révolution a des spécificités égyptiennes, c'est que l'articulation entre ces différents domaines dans la «ville monde » qu'est le Caire et la région du delta du Nil, est en train de participer à une esquisse du paysage naissant du mouvement oppositionnel sur une planète «mondialisée». Tout le monde a pu le constater du fait de la contagion rapide de la révolution Tunisienne de l'Égypte à l'Espagne en passant par la Grèce, Israël, les USA et d'autres.

3 En Égypte, jusqu'à la semaine du 18 au 25 novembre 2011, la révolution n'a plus pris le caractère spectaculaire des grandes manifestations politiques nationales qui ont abouti à 
la chute de Moubarak, même si les manifestations place Tahrir ont été plus qu'hebdomadaires. Mais la révolution du 25 janvier n'est pas finie. Bien au contraire. Elle s'est approfondie par la rupture morale avec l'armée qui a perdu toute légitimité non seulement pour les forces vives de la révolution mais aussi pour une large partie de la population. On est très loin du mois de février où les manifestants célébraient leur union avec l'armée. Cette rupture avec l'armée est aussi une rupture avec toute une idéologie et mythologie nationale issues de la période des indépendances. Mais il y a plus. Cette rupture, même si elle a une date politique qui l'a rendue visible à tous, était l'expression de bien d'autres ruptures, moins immédiatement apparentes, dans l'école, la médecine, le droit et la loi, la religion, qui, toutes, témoignent de transformations profondes. Cette révolution, malgré l'apparence trompeuse - voire frauduleuse - et surexposée, des premiers résultats des législatives, s'est poursuivie par une floraison infinie, mais moins visibles de loin, d'initiatives locales, professionnelles, culturelles, économiques ou politiques extrêmement variées dont le déterminant commun est la poursuite de l'apprentissage des premiers mois de la prise en main individuelle ou collective de sa vie par le peuple égyptien, comme je l'ai développé dans Carré Rouge ${ }^{1}$.

Ce long processus, où les femmes et les hommes de ce pays tentent de se réapproprier l'idée qu'ils se ressemblent et qu'ils peuvent réaliser collectivement les rêves qu'ils font ensemble, a commencé déjà il y a quelque temps et durera probablement des années.

Par son caractère urbain, sa diffusion par internet et sa durée, cette révolution nous montre que la planète oppositionnelle ne se réduit pas sur une face du globe à des révoltes condamnées à n'être que des émeutes rapides ou marginalisées suivies de longues périodes de répression et, sur une autre face, à des conflits de classes populaires relativement nanties, protégées et conformistes. Elle nous révèle la marche de l'humanité réunifiée en train de chercher une nouvelle citoyenneté commune face aux barbaries que laissent entrevoir les décours de la crise économique internationale.

C'est pour cela que la place Tahrir s'est trouvée au centre de l'attention des peuples du monde. Ce n'était pas par l'effet d'une quelconque curiosité exotique mais le premier révélateur, avec la place de la Kasbah à Tunis, de trente ans d'évolution économique et humaine de la planète qui nous étaient restées jusque-là invisibles ou sans signification. Le surgissement soudain de la place Tahrir a fonctionné comme un révélateur, par lequel nous apprenons à lire les transformations du monde, ce que porte la crise comme futurs possibles, mais aussi un miroir de ce que nous sommes devenus personnellement, en même temps que de ce dont nous sommes capables. Place Tahrir, nous nous regardons. Nous nous découvrons avec surprise et joie dans les torses nus de ces jeunes gens aux numéros de téléphone écrits sur le corps pour prévenir leurs familles en cas de décès, signifiant qu'ils préfèrent mourir libres plutôt que vivre à genoux ${ }^{2}$. Nous redécouvrons la noblesse et le courage d'une autre humanité.

7 Tahrir est un miroir magique car ses reflets ont un pouvoir de métamorphoses. Ce catalyseur a révélé par une farandole de places publiques occupées autour du globe les premiers pas d'une communauté nouvelle, d'une citoyenneté mondiale réinventée et libérée. Ce n'est pas un mince renversement que ce soit les Arabes, les peuples les plus méprisés en occident, qui portent le drapeau de ce que nous avons de meilleur en nous. 


\section{Démocratie parlementaire, dictature des " marchés ", dette égyptienne et pouvoir islamique}

C'est à l'aune de cette insurrection mondiale des consciences, que nous devons mesurer l'immense haine que les défenseurs de l'ordre ancien ont fait rentrer dans la fabrication frauduleuse des récents résultats électoraux en Tunisie, Égypte et Maroc ${ }^{3}$. La place Tahrir dévoile la marchandisation de la démocratie médiatico-parlementaire, dont l'idée est vendue comme un substitut à la joie de la démocratie directe oppositionnelle. Elle est un faux-semblant destiné, à éparpiller, à diviser la renaissance d'une conscience commune et mondiale, des exploités et des opprimés.

Parallèlement, la pseudo-démocratie parlementaire ne pourra pas prendre corps dans ces pays. Ils sont si pauvres qu'ils n'ont pas les moyens de l'accompagner de l'ensemble de ses particularités, une presse libre, la liberté de réunion et d'association y compris dans les usines, une justice indépendante, une école baignant toutes les classes sociales, un État laïc, mais surtout un niveau de vie autrement supérieur à celui d'aujourd'hui, qui lui permettrait d'exister et de durer un tant soit peu. C'est la démocratie directe des grèves et de la place Tahrir qui permet ce succédané actuel de marche vers la démocratie parlementaire. La question politique du moment se résume au cheminement de cette prise de conscience.

10 La période actuelle sera soit une transition entre la dictature discréditée de Moubarak et une autre, rafraîchie, soit le passage à une démocratie directe assumée. C'est pourquoi, tout en soutenant l'apparence d'un processus démocratique, les pays occidentaux veillent à rapprocher les élites militaires et patronales égyptiennes, des familles régnantes et des grands conglomérats du Golfe, toujours plus étroitement liés aux États occidentaux.

11 L'Arabie saoudite a promis 4 milliards de dollars à l'Égypte, plus que les montants promis par les États-Unis et l'Europe. La Kuwait Investment Authority a annoncé en avril qu'elle créait un fonds d'investissement souverain d'un milliard de dollars pour investir dans des entreprises égyptiennes. Le groupe koweitien Kharafi, dont on estime qu'il a déjà 7 milliards investis en Égypte, a annoncé qu'il empruntait 80 millions de dollars pour investir dans ce pays. On rapporte que le Qatar, aussi, envisagerait d'investir jusqu'à 10 milliards de dollars, selon son ambassadeur en Égypte.

12 Mais il y a plus. Actuellement, la dette extérieure de l'Égypte s'élève à environ 35 milliards de dollars. Entre 2000 et 2009, bien que le pays ait payé environ 24,6 milliards pour service de la dette le niveau de la dette égyptienne s'est accru d'environ $15 \%$. La différence entre les prêts reçus et les montants remboursés, s'est élevée à 3,4 milliards de dollars durant la même période. En d'autres termes il y a plus d'argent qui coule des pauvres d'Égypte vers les banques les plus riches d'Amérique du Nord et d'Europe, que l'inverse. Or, pour ces derniers, il faut que ce flux continue.

13 Voilà l'arrière-fond des discussions occidentales autour de l'Égypte et ce que cache le satisfecit donné par Obama au succès « islamiste » des élections.

14 Obama a été explicite. "L'objectif doit être un modèle dans lequel le protectionnisme cède le pas à l'ouverture [...]. L'appui de l'Amérique à la démocratie sera par conséquent basé sur la garantie de la stabilité financière, [...] l'intégration dans des marchés en concurrence les uns avec les autres et avec l'économie mondiale.» Il faudra donc: " $1^{\circ}$ Ouvrir des secteurs protégés [...] qui ont des barrières contre les investisseurs étrangers... 
$2^{\circ}$ réduire les taxes à l'importation et les barrières non tarifaires. $3^{\circ}$ lever la protection des entreprises étatiques en les exposant à la concurrence. »

Bref, aux côtés des investisseurs étrangers, la situation va offrir aux plus grands groupes patronaux d'Égypte (dont l'armée et les islamistes) d'énormes occasions de prendre des parts en propriété dans les principaux projets d'infrastructure et autres services publics privatisés. Le lien des islamistes avec l'Arabie saoudite et les pays du Golfe donnent aux capitalistes occidentaux la garantie que les Frères musulmans, les groupes salafistes en Égypte, Ennahda en Tunisie, sauront peser de tout leur poids dans cette politique de libéralisation.

C'est à cette fin que les porte-parole des Etats-Unis, de l'Europe et les médias à leur suite, insistent pour dire que les révolutions arabes n'étaient pas des révoltes contre plusieurs décennies de néo-libéralisme, mais seulement un mouvement contre un Etat qui avait mis des obstacles à la poursuite de l'intérêt individuel donc au marché libre. Le président de la Banque Mondiale, Robert Zoellick, faisant allusion à Mohammed Bouazizi, le jeune vendeur d'un marché tunisien qui s'est immolé par le feu et était devenu le catalyseur du soulèvement en Tunisie, déclarait : «Monsieur Bouazizi a au fond été poussé à se brûler parce qu'il était harcelé par des réglementations bureaucratiques ... » Ce serait donc faute d'un marché libre que le jeune homme s'est suicidé !

17 Mais ils risquent d'être surpris. Le processus en Égypte est une attaque contre les couches sociales privilégiées et ne peut pas être réduit à une question de «transition démocratique » ni d'élections contrôlées par le monde de l'argent. Nous n'en sommes qu'au début du processus révolutionnaire.

\section{Les raisons de la récente islamisation. La police des corps et des âmes}

18 Avec les premiers résultats des élections, les médias ont parlé d'une vague islamiste. Pour eux, les peuples, faute d'éducation et d'éducateurs, ne pourraient que se livrer à la barbarie.

19 Mais on ne peut penser ces résultats, l'islamisation, comme la dés-islamisation, sans comprendre la nature profondément policière de la société égyptienne. Avant la révolution du 25 janvier, le policier était un des symboles de l'injustice et de l'oppression. Se défaire de la police, puis contester le pouvoir de l'armée, c'est aussi faire un pas pour se défaire de bien d'autres valeurs, dont la religion.

20 La police égyptienne est un corps de 4 à 5 millions de personnes, jadis toute-puissante et aujourd'hui en décomposition. Cela explique en partie sa violence, elle qui lutte aujourd'hui pour sa survie avec un énorme sentiment de revanche vis-à-vis de la population après la révolution.

21 L'Égypte était un État basé sur la sécurité et la répression. Chaque institution publique avait le devoir de maintenir le pays sous pression constante. Et $80 \%$ des institutions publiques appartenaient à ce que l'on appelle l'« Assistance policière ». Ce ne sont pas des officiers de police, ni des militaires classiques mais des fonctionnaires qui travaillent au niveau de la rue, qu'ils occupent totalement ; ils sont partout, surveillent les mosquées, les banques, les hôpitaux, les écoles. Ces policiers de la rue ne recevaient que peu de formation, étaient payés une misère - 30 euros par mois - et ne coûtaient donc rien au 
régime. Ils se payaient sur le dos de la population. Ceux qui avaient la chance de "surveiller» un secteur clé, comme le bâtiment, ou les réseaux d'électricité ou de télécommunication, pouvaient rapidement devenir très riches grâce aux pots-de-vin.

Ces policiers viennent des milieux populaires, alors même qu'ils causent une grande souffrance à la population. Après la révolution, ils ne pouvaient continuer à intimider chaque citoyen pour trouver de l'argent. Le 23 février, douze jours seulement après le départ de Moubarak, ils ont donc déclenché une grève et ils ont mis le feu au ministère brûlant beaucoup de documents ce qui arrangeait tout le monde. Leur salaire a été augmenté, ils gagnent aujourd'hui entre 100 et 180 euros par mois. Ce qui leur permet de survivre. Les pots-de-vin existent encore, mais leur volume a diminué, et ce n'est plus un système.

Le fait que le deuxième soulèvement de la population ait eu lieu fin novembre est un symbole. Les mois de novembre et décembre étaient les pires. Beaucoup n'osaient plus sortir de chez eux, de peur d'être arrêtés. En effet, les deux derniers mois de l'année sont d'habitude les plus durs car les officiers de police tiennent à tout prix à clôturer les dossiers pour être promus.

Depuis février, les policiers ont donc organisé plusieurs manifestations un peu partout dans le pays, dont certaines furent très violentes. Les policiers de base se sont mis en grève illimitée à partir du 24/10 pour demander la démission du ministre de l'intérieur. C'est aussi un signe que l'armée reprend la place qu'elle avait avant Sadate, y compris au niveau des affaires et du volume des pots-de-vin, au détriment de la police, et que la relation entre la police et l'armée ne fonctionne pas très bien. L'armée, seule, est aux commandes du navire.

Cette police, si proche des milieux populaires, ne pouvait être supportée sinon acceptée, que par l'espoir d'un grand idéal commun, le nationalisme. L'élan de l'indépendance s'éloignant, il fallait mettre sous contrôle cette porte de sortie par l'esprit. La religion des mosquées pouvait y servir. Pas celle des confréries soufis si influentes en Égypte, mais trop « bohèmes » et d'esprit trop « libertaires ». Non, il fallait une religion profondément adaptée à cet univers policier, qui soit toute dans les formes, les règlements, n'existant que par les interdits. Et le principal d'entre eux, le contrôle des femmes qui transforme chaque homme en un policier intime et familial.

\section{L'âme d'un monde sans âme}

Les rythmes de la renaissance humaine en Égypte sont donnés par la rupture avec ce fond de relations construit autour de profondes transformations apportées par trente ans de mondialisation économique sur l'ensemble de la planète auxquels la société égyptienne n'a pas échappé...

L'univers social, culturel, psychique égyptien est profondément islamisé, mais, on aurait envie de dire en même temps, très superficiellement. L'islam a bien sûr une longue histoire dans ce pays. Mais l'islam dominant aujourd'hui est de facture très récente.

La jeunesse islamique attirée comme un aimant par la place Tahrir est en train de rompre avec les vieilles organisations et leurs discours. Le succès électoral «inattendu » des salafistes en Égypte en est peut-être un des signes les plus visibles. À l'heure où, du $18 \mathrm{au}$ 25 novembre, à l'occasion du deuxième soulèvement de la place Tahrir, se posait la question du choix d'une deuxième révolution contre l'armée, ou alors, de la participation 
au scrutin des législatives encadré par l'armée et les Frères Musulmans, ces derniers ont déserté la place au profit du vote. Par contre plusieurs groupes salafistes sont restés sur la place, solidaires des jeunes en lutte, même si c'était, pour certains, afin de convaincre la jeunesse la plus avancée de se rendre aux urnes. Le succès inattendu de dernière minute des salafistes trouve peut-être là, dans leur hostilité à l'armée, une de ses explications.

Bien des jeunes ne voient pas dans le salafisme le mouvement réactionnaire, voire fascisant, financé par l'Arabie Saoudite, soutenu en sous-main et infiltré par l'armée pour affaiblir les Frères Musulmans, mais un retour pur et honnête à la lettre de l'islam, humaine, sociale, révolutionnaire en dehors de tous les compromis institutionnels de la religion avec la dictature. C'est déjà sur ce fond de rupture institutionnelle de la religion avec le système, que sont nées, depuis la révolution du 25 janvier, plusieurs nouvelles organisations de jeunes issues des Frères musulmans.

Bien sûr, on peut être inquiets, à juste titre, de la capacité du mouvement salafiste à capter les aspirations d'une partie de la jeunesse. Leurs exactions contre les femmes, les coptes et les libertés, l'encouragement du résultat électoral à ce genre de pratiques, font froid dans le dos. Cependant, il ne faut pas seulement regarder ces événements par euxmêmes, mais comme un moment dans le décours d'une révolution qui s'approfondit en Égypte et, peu à peu, à l'échelle mondiale.

Ce sont les sociétés de Sadate et Moubarak qui ont fabriqué l'islamisation récente du pays. Avec la chute de Moubarak, l'édifice est seulement ébranlé, la religion guère touchée. Par contre avec la chute de l'armée et de tous les petits Moubarak que recherche la deuxième étape de cette révolution, ce sont les fondements de la religion qui sont remis en cause.

Les problèmes de Sadate et Moubarak étaient de faire face au discrédit progressif des courants et des idées indépendantistes. Les mythologies d'une économie forte et libératoire dans le cadre national, voire arabe avec la RAU ${ }^{4}$, s'effondraient. La vague de libéralisme sauvage qui a détruit les protections sociales d'État, poussait les peuples dans une franche hostilité à tout ce qui venait de l'occident. En même temps que des millions d'égyptiens trouvaient un travail dans les pays du Golfe enrichis par la manne pétrolière, les islamistes savaient donner un écho concret à ces plaintes et souffrances, en remplaçant la fierté nationale par son écho mythifié dans la religion.

Sadate et Moubarak se sont appuyé sur ce nouvel état d'esprit en y voyant la possibilité d'un détournement des aspirations et des colères populaires. Ils virent dans l'islam la possibilité d'introduire dans le psychisme de chacun leurs propres règles policières au travers d'une police des mœurs librement consentie. À partir de la deuxième moitié des années 1970, ils se mirent à islamiser la société tout en combattant les ambitions politiques des mouvements musulmans.

34 Ainsi dans la deuxième moitié des années 1970, les autorités, offrirent aux familles des aides financières pour chaque fille qu'elles voileraient. En 1980, ils firent de la charia le deuxième article de la constitution: "l'islam est la religion de l'État, la charia est la source de la législation ». Depuis 1985, chaque fois qu'une réforme est introduite dans le droit de la famille, il faut l'approbation du Mufti c'est-à-dire du chef du clergé. En 2006, les autorités rendirent obligatoire l'attribution d'une religion sur les cartes d'identité, musulman, chrétien ou juif. On naît musulman, on ne peut pas être athée ou bouddhiste et on ne peut pas renoncer à la religion musulmane sous peine de mort. Même si pour ce dernier point, la justice civile ne condamne qu'à la prison ou à des amendes pour « trouble à l'ordre public », si un imam quelconque au nom de l'interprétation religieuse 
de la charia, prononce la mort, n'importe quel illuminé de Dieu peut s'en prévaloir pour tuer l'apostat. C'est arrivé à un certain nombre d'intellectuels ou artistes.

L'Égypte a adopté le système juridique français, le code Napoléon, mais cela ne s'applique pas aux affaires de la famille qui dépendent de la «charia » et des codes des différentes communautés religieuses. Des tribunaux spéciaux de la famille sont censés veiller à leur application. Un époux peut interdire à sa femme de quitter l'Égypte par une déclaration administrative élémentaire. Il peut répudier sa femme simplement en le lui disant, même si celle-ci ne le veut pas. La polygamie est autorisée. Seuls les enfants musulmans peuvent hériter en cas de familles comportant enfants chrétiens et musulmans. En justice, la parole d'un musulman vaut celles de deux chrétiens, etc.

Mais cette islamisation de la société, qui rendait la vie des Égyptiens de plus en plus douloureuse et de moins en moins maîtrisée, s'est heurtée en même temps à des forces contradictoires.

\section{Révolutions urbaines, salariales et matrimoniales}

Depuis l'an 2000, l'épouse a un recours juridique possible contre la répudiation. Depuis cette date également, la répudiation pour les hommes est rendue plus difficile. En 2004, la réglementation des tribunaux de la famille a été un peu assouplie en même temps qu'en 2008, l'âge minimum du mariage est passé de 16 à 18 ans.

Ces évolutions juridiques trouvent leur fondement dans l'évolution de la société ellemême.

La crise de surproduction mondiale conduisant à la libéralisation économique de ces trente dernières années, a déplacé la géographie de la production vers certains pays du tiers-monde, en brisant le peu de protections étatiques issues des indépendances, et familiales ou tribales, de temps plus anciens. Elle a poussé les pauvres à chercher une vie meilleure dans les villes.

Le Caire est passé de 3 millions d'habitants dans les années 1960 à 20 millions aujourd'hui. Sanaa, capitale du Yémen, de 50000 en 1960 à 2500000 aujourd'hui. Dans le monde arabe en 1950, sur les 100 millions d'habitants, $26 \%$ vivaient en ville, aujourd'hui ils sont $66 \%$ pour les 350 millions actuels. Ces pays se sont couverts de quelques mégalopoles et d'une foule de villes moyennes et petites.

41 L'Égypte a une urbanisation et densité de population six fois plus importante que celle de la Hollande - la plus forte d'Europe - une population d'environ 85 millions d'habitants, très jeune, (âge médian de 24 ans), une classe ouvrière de 8 millions de salariés, avec le secteur industriel le plus développé du monde arabe et d'Afrique (la plus grosse usine textile d'Afrique et du Moyen Orient à Mahallah al Kubra, 24000 salariés chez Misr Filature et Tissage), et un secteur « informel» de petits boulots de 10 à 13 millions de « jobeurs».

Dans les années 1980-1990, la «libéralisation » de l'économie mondiale a entraîné la fermeture des entreprises d'État comme la destruction des services publics provoquant la croissance de la pauvreté d'un côté et de la richesse de l'autre. Et quand elle a implanté d'autres entreprises, plus récentes, elle l'a fait dans des conditions dégradées.

Les bidonvilles aux conditions de vie dramatiques, 1 à 4 millions dans celui du cimetière du Caire, la jungle urbaine et l'émigration bousculent les traditions, détruisent les vieilles 
solidarités mais aussi ce qu'il y a de plus pesant et coercitif dans la tradition et créent ainsi un "espace de liberté ». Cette "liberté » est celle d'un prolétariat féminin et enfantin à être exploité sans limites. Mais en même temps qu'elle devient cette jungle, la ville mixte les traditions et fait sortir ces nouveaux prolétaires de leurs anciennes solidarités pour leur en faire chercher de nouvelles dans ces « villes-monde ».

L'émigration massive des Égyptiens pour trouver du travail à l'étranger a poussé bien des femmes à travailler, et, par là, à trouver une certaine émancipation. Un véritable bouleversement souterrain a sapé les assises de ce retour militaire en « charia » de même que les bases des régimes dictatoriaux sclérosés fondés tous deux sur la famille patriarcale, le mariage en bas âge et entre cousins germains, la soumission des femmes et un taux de fécondité élevé. L'âge du mariage qui était de 17-18 ans pour les femmes est passé à 23 ans. Ce qui signifie un célibat plus long. La fécondité est passée de 6 à 7 enfants à environ 3. On estime le taux de contraception à près de $60 \%$. Le nombre d'avortements, encore interdits, explose. L'écart d'âge traditionnellement élevé entre époux diminue comme l'habitude du mariage endogame. La durée du mariage, jadis assez courte du fait des facilités de répudiation pour les hommes, s'allonge. La polygamie a quasiment disparu.

Les régimes politiques sclérosés se sont écroulés. On assiste à la même fissuration du système religieux qui prend pour le moment la forme de querelles de générations. Peu à peu, l'ancien ascendant religieux intériorisé, prend, dans cette société qui se libère, l'aspect d'un contrôle de plus en plus extérieur et pesant, de plus en plus hypocrite, tatillon et fragile que celui de la police.

L'appareil policier maintenait la terreur. Mais le gendarme n'était pas intériorisé dans le cerveau des hommes, comme il l'est souvent dans le monde occidental où des siècles d'oppression et d'exploitation capitaliste nous ont fait enfouir au plus profond de nous l'acceptation des règles d'un monde qui marche sur la tête: aller se faire exploiter tous les jours, toute la journée, toute l'année et à l'heure... Cette gendarmisation psychique s'est faite ces dernières décennies par la religion. En même temps que la libéralisation de l'économie amenait le gouvernement à abandonner les secteurs étatiques de l'économie au privé, les autorités abandonnaient bien des services publics, hôpitaux, écoles, services de ramassage des ordures aux islamistes. Nasser avait interdit officiellement les Frères Musulmans, mais le régime leur permis de focaliser leurs activités sur les élections des unions estudiantines, des clubs des professeurs universitaires et des syndicats durant les années 1970-1980, puis de disputer les élections de l'ordre des Médecins pour la première fois en 1984 pour en conquérir la majorité en 1992, avant d'investir les syndicats des Ingénieurs et des Pharmaciens.

\section{Sous le vote, d'autres votes contre tous les petits Moubarak militaires, civils ou religieux}

Les islamistes ont utilisé leur présence sociale, acquise dans la période précédente, lors de la campagne électorale des législatives, en multipliant les actes de charité, par des soins et des médicaments gratuits, des cours à domicile bénévoles, des produits alimentaires de base distribués gratuitement ou moins chers, notamment la viande complaisamment fournie par les fermes possédées par les militaires, la prise en main du ramassage des ordures que les services publics dégradés ont quasiment abandonnés voire détruits, par 
exemple lors de la répression des chiffonniers coptes qui en avaient le quasi-monopole au Caire, par l'organisation de la circulation automobile en ville devenue épique depuis la fuite des policiers ou leur état quasiment permanent de grève depuis des mois...

D'un autre côté, un des effets de la révolution a été la multiplication des grèves dans toutes les professions, avec des revendications économiques, salariales, de conditions de travail mais aussi des revendications politiques, et surtout l'exigence de la démission des petits Moubarak à tous les niveaux de la société et donc aussi dans les associations et syndicats de l'époque du dictateur.

49 Une nouvelle confédération et plus de 150 syndicats ouvriers libres ont été créés regroupant plus de 1700000 salariés. Un syndicat de la main d'oeuvre féminine agricole a été bâti pour demander notamment l'égalité de traitement avec les hommes dans un secteur où travaillent 4 millions de femmes et où il leur était interdit jusque-là de s'organiser.

50 La perte d'influence des islamistes a été extrêmement rapide dans les associations professionnelles pré-existantes puisque, lors des dernières élections, cet automne, ils y ont perdu la majorité chez les médecins (et même 70 \% des sièges dans les grandes villes) et les journalistes et pourraient bien perdre un peu de leur crédit chez les avocats et les ingénieurs, de même qu'ils sont bousculés chez les professeurs d'université, les étudiants ou les artistes par de nombreuses associations naissantes. Ils ont gardé leur majorité chez les enseignants, bien que cela puisse changer rapidement, car les Frères musulmans viennent de s'opposer à la dernière grève de cette profession en septembre, la première depuis 1951, appelée par leur nouveau syndicat et suivie par près de $70 \%$ des enseignants.

51 C'était déjà en s'opposant à la grève des médecins - la première dans l'histoire du pays suivie à $90 \%$ (les médecins de base hors du Caire gagnent guère plus de 30 euros par mois) qu'ils avaient déjà perdu leur influence. Un comité de grève nationale a été créé. Les Frères Musulmans affirmant que la demande de démission du ministre de la santé était « irréaliste », ils ont lâché le mouvement provoquant la division dans leurs rangs, certains membres des Frères votant malgré tout pour la grève.

52 C'est dans cette même période et avec ce même état d'esprit qu'à l'hôpital Minchiyet AlBakri au Caire, les différentes catégories de personnel ont décidé de construire un syndicat libre commun dépassant les corporatismes et ont élu les plus jeunes d'entre eux à sa direction. Dans la foulée, les salariés ont décidé de changer de directeur de l'hôpital en procédant à l'élection de cette fonction. Ils ont informé la sécurité pour qu'elle ne laisse plus entrer l'ancien directeur. De peur de troubles, le ministère a accepté le nouveau directeur élu. Il y a aujourd'hui quatre hôpitaux où des syndicats libres sont créés, et des dizaines d'autres en voie de création.

53 Le conseil syndical actuel des journalistes élu en novembre est le premier conseil élu après la révolution du 25 janvier. Il ne cherche pas à faire plaisir au pouvoir alors que plusieurs journalistes ont été dernièrement convoqués devant le Parquet militaire pour justifier leurs propos oraux ou écrits et que, depuis 1996, ils peuvent être mis en détention provisoire pour leurs écrits.

Deux publications viennent d'être censurées pour avoir traité des sujets «portant atteinte aux intérêts nationaux » et les bureaux d'au moins trois chaînes privées ont été pris d'assaut par des militaires. La nouvelle majorité syndicale vient en effet, comme les nouveaux syndicats ouvriers, de décider de soutenir et encourager ses membres à refuser de se présenter devant les cours militaires, s'ils y étaient convoqués. Ce refus des procès 
militaires pour les civils - plus de 12000 personnes y ont déjà été brutalement condamnées ces derniers mois- est une des exigences de la population qui fut à l'origine du soulèvement de novembre contre le pouvoir de l'armée. Le syndicat a décidé de soutenir tout citoyen civil convoqué devant les cours militaires, en allant jusqu'à utiliser leurs journaux pour cela.

Mais les tribunaux civils pourraient bien subir rapidement un tel discrédit.

Un certain nombre de juges étaient aux ordres du pouvoir. Le jugement du procès de la fameuse «bataille des chameaux » de la place Tahrir mettant en cause journalistes de l'ancien régime, ministres et hauts militaires, a été reporté comme celui de l'ex-président Hosni Moubarak, car les avocats des familles des victimes ont demandé le remplacement du président du tribunal, l'accusant de conduire les audiences d'une manière «non conforme à la justice ».

Cette lutte contre les petits Moubarak dans la Justice est à la base des conflits et des bagarres physiques actuels très violents entre juges et avocats, pourtant censés représenter dans le pays la pondération, la loi et le droit. Les juges veulent pouvoir infliger jusqu'à cinq ans de prison aux avocats qui auraient insulté un tribunal. Les avocats demandent la révocation des juges compromis avec l'ancien régime et refusent de se présenter dans leurs audiences. Ils demandent également que ces juges soient démis de leurs fonctions de présidents des bureaux de vote pour veiller à la régularité des scrutins. Depuis des mois le système judiciaire civil est paralysé.

Dans le syndicat des ingénieurs, 475000 membres, dirigé par les Frères Musulmans depuis 16 ans, les jeunes n'ont pas d'ambitions démesurées par rapport à cette emprise, mais ils viennent de créer une nouvelle association, le Marsad, pour que « le syndicat devienne un outil révolutionnaire pour réaliser les demandes des travailleurs » et pas seulement d'assistance auprès de ses membres, comme jusqu'à présent.

\section{Les grèves de l'automne changent de nature}

Après deux vagues de grèves, l'une de 3 jours en février 2011 qui a fait tomber Moubarak, une deuxième au printemps dans la foulée de la chute du dictateur, beaucoup ont vu dans la vague de grèves qui a traversé le pays en septembre-octobre une seconde révolution, socio-économique cette fois-ci, ou de la faim comme ont dit bien des participants. Sans aller jusqu'à l'idée d'une deuxième révolution, ce mouvement, d'une ampleur inégalée, a déstabilisé le pouvoir en place, mis en cause la légitimité de l'armée à la tête du pays et donné l'élan et les bases sociales du soulèvement de novembre place Tahrir. Il s'agit d'une nouvelle phase dans le processus révolutionnaire, qui modifie le caractère du mouvement par en bas.

On a assisté en effet à une recrudescence sans précédent de protestations, grèves et sit-in dans plusieurs secteurs économiques. Instituteurs, médecins, ingénieurs, infirmières, avocats mais aussi employés des transports publics, des postes, des banques, des hôtels, des musées, des ministères, des télécom, des chantiers archéologiques, des clubs de sport, des collectivités urbaines, du métro, dockers, contrôleurs aériens, agents d'aéroports, hôtesses de l'air, pilotes, garagistes, ouvriers agricoles, travailleurs intérimaires, chauffeurs de taxi, vétérinaires, pécheurs, agents de sécurité, marins, étudiants, ambulanciers, mineurs, cheminots, paysans, ouvriers du bâtiment, des travaux publics, du textile, du pétrole, du plastique, de l'industrie pharmaceutique, du sucre, de l'acier, des 
machines agricoles, des usines militaires, et de bien d'autres secteurs encore, sans oublier les policiers de base ou les salariés des mosquées.

61 On compte parmi elles des grèves nationales massives: des centaines de milliers d'enseignants ont cessé le travail, 30000 travailleurs de l'industrie sucrière ont fait grève trois semaines. Pendant douze jours, 70000 travailleurs des autobus du Caire ont fait grève, et les bus ont été utilisés pour bloquer les rues autour du Parlement. Ces grèves ne sont pas simplement importantes à cause du nombre élevé de participants. Elles le sont également en raison de leur degré de coordination au niveau régional, sectoriel et national, ainsi que par le type de revendications mises en avant par les travailleurs: hausse des salaires et primes, meilleures conditions de travail, meilleure formation, réforme du secteur concerné, amélioration du secteur public, mais aussi comme un leitmotiv, la démission de tous les petits Moubarak à tous les niveaux et la fin du pouvoir des militaires.

62 Le gouvernement, pour éviter que cette généralisation des mouvements ne se transforme en grève générale où les ouvriers auraient pu prendre conscience de leurs intérêts collectifs et politiques, cède à de nombreuses revendications d'un certain nombre de professions, pour que d'autres, isolées, débouchent sur des impasses temporaires, comme celle des enseignants. L'ampleur du mouvement de protestation était en effet particulièrement significative au sein du corps professoral avec en tête de leurs revendications l'augmentation du budget de l'Éducation nationale pour l'avenir des enfants, le renouvellement des cadres du ministère de l'Éducation, les estimant largement corrompus et la coordination des nouveaux syndicats et des mouvements des jeunes. Même chose pour les salariés de Telecom Egypt qui, bloquant 60 centraux sur 72, revendiquaient la dissolution du conseil d'administration, accusé de corruption, la démission du directeur général, la libération de 5 collègues condamnés en prison pour tentative d'assassinat sur le directeur et enfin l'ouverture des livres de compte afin de faire le point sur les revenus, les dépenses et autres opérations financières.

Le gouvernement a par contre cédé aux chauffeurs de bus des transports publics du Caire mais surtout aux ouvriers de l'usine textile de Mehalla Al Kubra qui ont joué un rôle central dans tous les mouvements depuis 2006 et qui menaçaient en octobre d'appeler à la grève générale. Les ouvriers du secteur public du textile sont désormais organisés dans une seule entité.

Ce qui n'est pas indépendant du fait que le tribunal administratif ait décidé de renationaliser trois entreprises textiles passées précédemment au privé, les sociétés Tanta, Misr Chébine Al-Kom et Al-Nasr. Il faut dire que les ouvriers de la société de Chébine Al-Kom ont, à eux seuls, organisé 128 manifestations depuis octobre 2005, date de la privatisation de leur société. La décision du tribunal a créé un effet domino. Les ouvriers du public ont quelques avantages qui n'existent pas dans le privé. Aussi les salariés de 9 autres sociétés, mais peut-être aussi des secteurs entiers du fer, de l'acier et de la céramique, ont-ils réclamé le retour dans le public.

La nouvelle coordination ouvrière du textile s'accompagne du regroupement des professeurs, des employés de la poste, des médecins, des ouvriers des sociétés publiques du sucre.

66 L'audace des grèves est une source d'inspiration. Des patrons sont séquestrés, des usines occupées, y compris par des chômeurs, voire redémarrées sans leurs directeurs. Les travailleurs commencent à penser à un monde sans contrats de travail temporaires; avec 
un niveau de salaire permettant une redistribution des richesses et où on ne se contenterait pas de résister aux privatisations mais où auraient lieu des renationalisations. Il est fréquent par ailleurs que les comités de grève et les négociateurs soient élus directement par le personnel en grève. L'habitude a été également prise de rendre compte directement des résultats des négociations en assemblée générale. Cette démocratie directe par en bas est une fusion des aspects sociaux et démocratiques de la révolution. De là, peuvent surgir des organisations démocratiques de masse émergeant par le bas, source d'organes de pouvoir alternatif sur le plan politique et social, conscients de la nécessité d'une deuxième révolution pour briser les vieilles structures de l'Etat.

Les principaux partis islamistes et libéraux sont terrifiés par les grèves et y sont résolument opposés en s'aplatissant constamment devant le Conseil militaire.

Particulièrement visés, les généraux ont riposté avec une brutalité croissante. Les autorités militaires et les secteurs décisifs du capitalisme égyptien ont la volonté de mettre fin à la combinaison qui tend à s'établir entre revendications sociales et démocratiques. Cela ne date pas d'aujourd'hui. Depuis le 26 mars, l'armée a décidé qu'il sera désormais interdit de se mettre en grève et de manifester. Il est interdit également de constituer des partis sur une base de classe et de se présenter aux élections sous l'étiquette « ouvrière » sans voir le label du syndicat officiel du temps de Moubarak.

Le 10 septembre, le Conseil suprême des forces armées (CSFA) réaffirmait la nécessité de prolonger l'application des lois d'urgence. Il le fit après une "attaque » organisée en sous-main par ses soins, contre l'ambassade d'Israël en date du 9 septembre. Le 12 septembre, le major-général Mahmoud Shahin, déclarait : «Les violations répétées de la sécurité publique ont contraint le CSFA de faire appel, à nouveau, aux mesures prévues par l'état d'urgence. Ces événements incluent des actes de gangstérisme, de terrorisme, de trafic de drogue, de sabotage, d'interruption de la circulation et de blocage de routes ainsi que la diffusion de fausses rumeurs et fausses informations. " Donc des cours martiales doivent intervenir pour « lutter contre la vague de crimes menaçant la sécurité nationale et le déferlement $d u$ chaos ». La reprise en main par le pouvoir militaire est clairement proclamée.

70 Mais en même temps que les grèves, sans lien direct mais parallèlement, le 16 septembre, la place Tahrir a connu une nouvelle mobilisation, avec une forte participation de paysans pauvres, sous le mot d'ordre: "Non à la loi d'urgence", et la revendication d'une accélération de la transition du pouvoir militaire vers un pouvoir civil. Le 9 octobre, les militaires montent une provocation contre une manifestation copte, en tuent une vingtaine et tentent de dresser les musulmans contre les chrétiens. La situation se retourne contre eux, les manifestants coptes et chrétiens s'unissent contre l'armée. C'est un fait plus exceptionnel encore que les slogans visant Moubarak. L'armée en Egypte est une chose sacrée, intouchable. C'est un tournant. L'image de ces jeunes manifestants offrant des roses aux militaires postés sur leurs chars aux alentours de la place Tahrir n'est plus qu'un souvenir. Les mêmes manifestants sur la même place n'hésitent plus à crier : «A bas le règne des militaires ». Des manifestations vont se succéder jusqu'au 25 novembre. L'armée recule et cède, en parole, sur les tribunaux militaires pour les civils, un président élu...

71 Les élections, législatives, sénatoriales et présidentielles qui débutent le 28 novembre et qui devraient s'étendre jusqu'à la fin du printemps 2012, malgré les appels au boycott des jeunes et la faible participation, sauvent les généraux. Le succès islamiste, comme meilleur espoir des généraux de création d'une légitimité démocratique de façade 
pourrait permettre au nouveau régime de contrôler, voire écraser les grèves et les manifestations. La polarisation voulue des élections entre les laïcs et les religieux, permet à l'armée d'exploiter le résultat électoral pour tenter de sortir de cette phase de transition avec un maximum d'indépendance politique et financière vis-à-vis de l'autorité civile élue. Une ambition ouvertement exprimée par le général Mahmoud Shahin.

\section{L'occupation des places, comme perception visible de la réappropriation de sa propre vie}

72 La place Tahrir, remplaçant les partis, a donné un visage et une tête, mais pas une direction, à ces révolutions qui traversent tout le monde arabe et ébranlent ses régimes dans leurs fondements.

On y voit de simples citoyens non politisés venant avancer leurs petites demandes personnelles qui tournent, la plupart du temps, autour de besoins fondamentaux : travail, nourriture, habitation, salaire. C'est là qu'est née la Coalition des jeunes Nubiens, 3 millions de personnes du sud de l'Égypte à structure proche du matriarcat, dont la majorité a été expulsée dans des conditions inhumaines lors de la construction du barrage d'Assouan.

74 C'est place Tahrir que des dizaines de millions de téléspectateurs égyptiens et arabes éberlués ont vu ce qui n'est pas anodin en Égypte, une cohabitation sans problèmes, jour et nuit, des hommes et des femmes, montrant que ces archaïsmes ne sont pas inscrits au plus profond de la «nature humaine » mais ne tiennent que par ces régimes dictatoriaux qui y trouvent leurs assises. Cette cohabitation a révélé aux Égyptiens, la profondeur de leurs propres évolutions qu'ils avaient vécues ces dernières décennies sans en prendre vraiment conscience. La place Tahrir est comme un miroir que les autorités devaient absolument brouiller par des agressions sexuelles. C'est pourquoi on assiste à un déchaînement de haine et de violence faite aux femmes ces derniers mois comme un concentré du changement en cours. L'armée demande des certificats de virginité et considère les manifestantes célibataires incapables d'en fournir comme des prostituées. Des groupes salafistes, agressent violemment les manifestantes, les battent et les insultent.

75 L'écrivain féministe et psychiatre égyptienne Nawal El Saadawi - auteur de trente ouvrages traduits en vingt-huit langues - occupante assidue de la place Tahrir, racontait il y a peu qu'elle était encore interdite de parole sur tous les grands médias télévisés, radiophoniques ou de la presse écrite égyptienne alors qu'elle s'exprimait librement dans bien des journaux du monde. Par contre tous les jours, les religieux ont sur les médias privés mais aussi publics, des heures et des heures de prêche, répétant les uns après les autres que les femmes doivent savoir garder leur place, à la maison.

76 C'est la raison pour laquelle l'armée a fait évacuer violemment la place Tahrir le 9 mars, puis qu'elle a été occupée sporadiquement par les partis mais que celle-ci est maintenant réoccupée depuis le 18 novembre comme symbole d'une prise de conscience plus forte. 


\section{C'est de la vie quotidienne que les choses changent et se construisent}

77 L'importance politique électorale des partis islamistes est inversement proportionnelle à leur perte d'influence dans le mouvement social. Il existe un conflit latent entre une importance politique certaine des partis islamistes et un éloignement, progressif et contrasté, mais réel, d'une partie de la population de l'emprise des normes religieuses sur la vie sociale et politique.

78 Les Égyptiens en ont assez des discours vides du style « la religion ou la démocratie sont la solution ». Un sondage mi-octobre donnait seulement $1 \%$ de la population pour considérer la démocratie comme le problème le plus important du moment alors que $62 \%$ pensaient que les problèmes économiques étaient les plus importants et souhaitaient à $76 \%$ un plus grand contrôle de l'économie par l'État. Seulement $48 \%$ des Égyptiens se sont déplacés pour voter alors que le vote est obligatoire (en tous cas, l'armée en fait courir la rumeur) et qu'on risquerait une amende équivalente à plus d'un mois de salaire moyen si on se soustrait à cette obligation. Et cela malgré un battage inimaginable pour voter. Certains craignent que la place Tahrir ne soit isolée du reste de l'Égypte. Elle est certes en avance, mais appartient dorénavant à chacun, faisant partie de l'insurrection des consciences qui se fait.

\section{Le foot en révolution}

Le scrutin électoral imposait au moins une candidate féminine. De fait, les femmes ont été reléguées au dernier rang des listes. Par contre, place Tahrir ou dans les grèves, les femmes sont bien présentes. En se posant le problème de leur protection, on voit bien souvent maintenant des services d'ordre protégeant aussi bien les manifestations que les femmes. Selon le ministre actuel de la Justice, le nombre de baltaguis (voyous au service du pouvoir) est évalué aujourd'hui à un demi-million, qui gagneraient de 500 à 5000 livres égyptiennes pour une seule mission. Les nécessités de se protéger des violences des voyous et des policiers comme de certains groupes salafistes ont amené les "Ultras", associations de supporters de club de foot, comme «Les chevaliers blancs » de Zamalek, habitués depuis longtemps aux combats de rue contre la police, à servir de bras armé de la révolution et de service d'ordre des manifestations. Souvent méprisés, voire craints par la population, assimilés parfois à des voyous, ces «Ultras» sont en passe de devenir des "gavroches» de la révolution. Des supporters de foot servant de protection aux manifestations féminines. Ne peut-on pas imaginer de plus belle illustration de cette insurrection des consciences?

Les premiers groupes "Ultras" sont apparus en Égypte en 2005. Ils critiquent les structures sportives patriarcales et autoritaires du Parti national démocratique (PND) d'Hosni Moubarak. Ils deviennent vite la bête noire des médias sportifs, qui les décrivent comme des voyous communistes, des athées, des drogués et, à l'occasion, comme des " déviants sexuels".

81 Ce mouvement, concomitant de la vague de grèves qui touche l'Egypte à partir de 2006, prend rapidement une ampleur inédite qui inquiète les services du raïs. Les «Ultras » se révèlent en effet capables de rassembler autour d'un noyau dur, des milliers de membres 
d'une vingtaine d'années qui se réunissent régulièrement, disposent de forums Internet et d'un budget autonome financé par les fans. Pour se protéger, ils utilisent des noms d'emprunt, développent des structures légères et secrètes. Ils n'hésitent pas à se rebeller violemment contre les humiliations et la brutalité de la police. Dès 2007, ils entrent en confrontation ouverte avec le ministère de l'intérieur. Les affrontements se multiplient dans les stades, mais surtout dans les rues à l'occasion de véritables batailles rangées. La police commence à arrêter les leaders chez eux, avant les matches, et les fait comparaître devant des tribunaux militaires. Certains sont battus et torturés. Comme le dit l'un d'entre eux : «Les flics, c'est ma passion, je les hais à en mourir. »

$\mathrm{Au}$ cours de ces affrontements, les «Ultras » développent des techniques de combat de rue dont l'efficacité éclatera au grand jour lors de la révolution. Ils sont les seuls à savoir mener des batailles rangées contre les forces de l'ordre. On les retrouve en première ligne sur tous les fronts : habitués aux gaz lacrymogènes, aux balles en caoutchouc et à franchir les murs du stade, ils savent renvoyer une grenade, sauter sur un camion de police, escalader les lampadaires et jouent un rôle logistique décisif dans les victoires des révolutionnaires. Créatifs, ils donnent aussi à la révolution ses slogans et ses rythmes emblématiques, typiques du répertoire des supporters. Cet héroïsme leur a valu une reconnaissance certaine parmi les activistes et l'admiration d'une grande partie de la jeunesse égyptienne. Mélange de fraternité romantique et d'individualisme forcené, leur style de vie provocant et déjanté impressionne une génération aux prises avec des carcans sociaux et religieux pesants. Leurs mots d'ordre et leurs emblèmes sont devenus les symboles d'une jeunesse qui a soif de rébellion. L'acronyme ACAB pour «All Cops Are Bastards » (« Tous les flics sont des bâtards ») parsème aujourd'hui les rues du Caire. Leur art de l'insulte font les délices des militants et leurs chansons sont désormais une part incontournable d'une culture underground en plein essor.

Les Chevaliers Blancs, les Ahlawy, et les Dragons bleus (les trois groupes les plus importants) réunissent près de 20000 membres actifs et sont susceptibles de mobiliser plus de 50000 personnes. Les Chevaliers Blancs disent qu'ils peuvent faire descendre 25000 personnes prêtes à combattre dans la rue en quelques minutes. Ce qui n'est pas loin d'avoir été vérifié dans les émeutes de novembre.

Les «Ultras» cultivent un anarchisme farouche soucieux de préserver leur grande diversité sociale qui les fait rassembler riches et pauvres, laïcs et islamistes, gauchistes, salafistes, libéraux et Frères Musulmans. Tous les matches sont des occasions privilégiées de ridiculiser les policiers en leur rappelant leur défaite du 28 janvier par des chants déjà cultes : « Corbeau stupide/T’étais nul en classe/T'as eu 10 sur 20 en payant un pot-de-vin/ Mais t'as quand même pu t'offrir les meilleures facs/Pourquoi tu niches dans ma vie ?/ Juste pour me la pourrir/On n'oublie pas Tahrir, fils de pute. » Une journaliste décrivait ainsi ces ultras : «Cette génération née sous Moubarak et avec Internet a été capable de créer une nouvelle identité contre la culture de la classe moyenne et la moralité ambiante. Ils sont une face de la révolution celle de la rage, de la colère. Pas la face proprette à fleurs du jeune poli : la face anti-famille, anti-institution, anti-morale, antireligion. L'armée a envisagé de faire jouer les matchs dans l'enceinte des casernes.

Plus la révolution s'avance vers une confrontation avec l'armée, plus les "Ultras " prennent de l'importance. Athlétiques, théâtraux, rapides, à demi dénudés avec leurs numéros de téléphone tatoués sur le corps, ils continuent à crier leur haine de la police et des militaires en enchainnant leurs chorégraphies fétiches, sous l'oeil réprobateur des Frères Musulmans, des salafistes et de certaines mères de famille. 


\section{L'art des rues au service de l'expression libre} depuis octobre pour expliquer le déroulement des élections et comment voter. De fait, l'échange se fait dans les deux sens. Les jeunes, souvent étudiants, apprennent à connaître les problèmes et à recenser les besoins. Ils ont découvert que le gouvernorat d'Assiout manque d'établissements hospitaliers. À Assouan, la question de la marginalisation des Nubiens pose problème. La campagne Ally sotak est prolongée par Ehmy sotak (protège ta voix). L'objectif est de suivre et contrôler le candidat tout au long de son futur mandat. Les jeunes de Ehmy Sotak créent des comités populaires chargés de constater si les habitants sont satisfaits de leurs candidats et de leurs projets et surtout s'ils ont réglé les différents problèmes qui minent leur circonscription.

90

Il y a aussi l'initiative Emsek feloul (arrêtez les partisans de l'ancien régime) qui vise à barrer la route aux symboles du régime de Moubarak. Une liste noire des noms des candidats «feloul» aux législatives est publiée sur internet. Une chanson populaire envahit les ondes. 2000 candidats PND se sont déjà retirés de la course électorale. Mais le travail n'est pas terminé : près de $70 \%$ des partis politiques continuent à présenter des candidats ayant appartenu au PND. 
91 Autre initiative : les comités populaires d'Alexandrie ont décidé de lancer une campagne de propreté baptisée «Le premier colis pour le gouverneur", en lançant les sacs de poubelles devant le siège de la municipalité :«A chaque fois que vous ne ramasserez pas les ordures des quartiers pauvres, tous les cadeaux de déchets vous seront envoyés sur votre lieu de travail ».

\section{Dans le vide laissé par la police et la religion, entre gangs, loi du talion et pollution} boueuses où les pieds s'enlisent. L'odeur des ordures qui s'entassent partout rend
l'atmosphère irrespirable. Les gens ont les nerfs à fleur de peau: les disputes et les l'atmosphère irrespirable. Les gens ont les nerfs à fleur de peau: les disputes et les
bagarres, parfois mortelles, font partie du quotidien. Des enfants, dont l'âge ne dépasse pas 12 ans, conduisent des toc-tocs (espèces de rickshaws à moteurs indiens) à toute vitesse, sans aucun respect du code de la route. Ils sont une proie facile pour les baltaguis. Les vols de ces véhicules, particulièrement faciles à revendre, connaissent une envolée record. Autour de Nahiya, les baltaguis avaient imposé leurs lois. Ils obligent les chauffeurs de toc-tocs à payer une redevance de 3 L.E. et ceux des microbus 5 L.E. pour avoir le droit de travailler. Dans ce quartier, la drogue est vendue en plein jour et la prostitution prolifère.

Face à la loi de la jungle les habitants se sont d'abord armés. Des chômeurs ont attaqué la station de police, pris les armes et roué de coups le commissaire de police. Les forgerons fabriquent toutes sortes de pistolets comme le sonore-gun, des pistolets à balles réelles ou à billes et même avec des balles en caoutchouc. Les femmes, avec l'électricien du coin, fabriquent un bâton électrique. Puis ils ont décidé de faire respecter la loi par euxmêmes. Le cheikh promulgue une fatwa, à l'encontre de la charia très respectueuse des institutions, qui affirme que les parents des martyrs de la révolution ont le droit de prendre leur revanche si la justice fait défaut. Plusieurs centaines de citoyens ont arrêté 4 voleurs de toc-tocs. Ils ont tué le premier en le traînant dans la rue, attaché à une corde. Puis ils ont coupé la main du deuxième et crevé l'œil du troisième.

L'horreur a fait les gros titres de la presse : «victimes de l'anarchie et de la baltaga ». Le but conscient ou non, est de propager la peur et l'idée diffuse que ces actes sont le fruit de la révolution. Le groupe des promoteurs de la déception, part de ce genre d'événements pour ne cesser de se lamenter sur la désolation qui sévit dans le pays, sur l'insécurité et le manque de civisme qui va jusqu'à pousser les enseignants, les médecins, les ouvriers et les employés du transport à réclamer plus de droits au détriment de l'économie et de la stabilité. Cependant, le citoyen honnête sait que le quotidien de sa famille a changé de manière positive depuis la révolution. Son frère a obtenu un poste dans un institut dépendant d'Al-Azhar après des années d'attente. Lui-même a renouvelé sa carte grise sans subir le parcours du combattant habituel, et les paysans de son village se réjouissent de la nouvelle attitude des fonctionnaires du ministère de l'Agriculture. 

Gurgaon, les siècles se côtoient et se heurtent à une vitesse et une dimension jamais connues, se raccourcissant à quelques décennies. Nous franchissons les temps, passant de l'ère des tribus à celle d'Internet ou de la mondialisation et des villes tentaculaires avec ses entreprises multinationales gigantesques, ramassant en quelques années le XVIIe siècle européen, 1789, 1848 entre février et juin, 1905, mais aussi 1968. Dans ce cadre, la logique de la prise en main de leur vie par les Égyptiens part du concret, de leur vie quotidienne par la démocratie directe contre un cadre institutionnel légué par l'histoire, l'armée, la police, les partis, la religion, la propriété des moyens de productions, le salariat, le monde de l'argent et du capital.

même temps que le monde n'a jamais été aussi petit pour les capitaux, jamais il n'a été autant mis d'entraves à la circulation des pauvres, faisant de leurs propres pays et des idéologies nationales de vastes prisons où ils sont condamnés à survivre ou mourir. En 
Égypte, la pauvreté est passée de 39 \% en 1990 à 48 \% en 1999 dans les régions urbaines et de 39 \% à 55 \% sur la même période dans les régions rurales. En 1999, la moitié de la population vivait déjà dans la pauvreté. 32 millions d'égyptiens sur 64 millions à l'époque étaient considérés comme pauvres et dans ces 32 millions, 6 étaient considérés comme très pauvres. Aujourd'hui, c'est pire. C'est pourquoi, 22 millions d'arabes ont émigré, souvent dans les pays du Golfe mais aussi en Europe ou encore plus loin. Dans le désespoir qui frappe ces pays, il n'y avait qu'une échappatoire : fuir à l'étranger, y travailler, faire des études, partir, rêver d'un ailleurs meilleur. Mais une bonne partie des émigrés dans les États du Golfe est revenue. Quant aux frontières européennes, elles sont de plus en plus hermétiques... Ce qui n'a pas été pour rien dans les soulèvements actuels.

Condamnés à vivre dans leur prison, à ne plus fuir, ni dans l'espace ni dans les rêves, les Égyptiens n'ayant plus rien à perdre, prennent en main leur vie et nous montrent le chemin de notre propre émancipation dans cette économie mondialisée, cette planète réduite par internet et où nos «acquis» fondant comme neige au soleil font fondre également les frontières qui empêchaient la renaissance d'un nouveau prolétariat mondial.

\section{Post-scriptum (le 21 décembre 2011)}

Hier mardi, l'après-midi avait commencé, comme depuis plus d'une semaine, par de nouveaux affrontements violents entre manifestants et forces de l'ordre autour de la place Tahrir, deux manifestations d'étudiants contre les violences policières et une manifestation d'ouvriers du ciment. La presse donne le plus fréquemment à ce jour depuis le début des affrontements de décembre, au moins 14 morts et plus de 800 blessés.

En fin d'après-midi une manifestation de femmes contre les violences policières commencée à 1500 ou 2000 a grossi au fur et à mesure qu'elle avançait pour atteindre entre 10 et 15000 personnes selon la presse (50 000 selon les facebookers les plus optimistes), des femmes de toutes conditions, de tous âges, voilées ou non voilées. Ce sont les violences faites aux manifestants, aux femmes en particulier (un test de virginité est demandé aux manifestantes sous peine de tomber comme prostituées) mais surtout l'image de la « jeune femme au soutien gorge bleu » (ne connaissant pas son nom, elle est appelée comme ça) dénudée et brutalisée par les forces de sécurité qui a mis le feu aux poudres. En plus des vidéos sur internet, le journal Al Tahrir avait titré avec cette photo en première page, et beaucoup de manifestantes portaient cette photo en pancarte. La manifestation était très dynamique, les femmes très heureuses, il y avait foule aux balcons et aux fenêtres qui applaudissait et criait son soutien pendant que les manifestantes demandaient à tout le monde de descendre dans la rue. Des hommes ont fait un service d'ordre tout le long de la manifestation pendant que beaucoup d'autres sur les trottoirs applaudissaient. Les slogans les plus repris étaient les « femmes d'Egypte sont la ligne rouge » (à ne pas dépasser sinon...) et «A bas le régime militaire ». Beaucoup de pancartes individuelles contre les violences faites aux femmes, avec sérieux ou humour, bref la colère et la joie.

106 C'est la première manifestation féminine de cette importance, à ma connaissance, dans l'histoire de l'Egypte. Pour comparer, le 8 mars 2011, on comptait quelques centaines de manifestantes vite dispersées par les violences des voyous et des salafistes exigeant qu'elles retournent à la cuisine. Le harcèlement sexuel est une réalité quotidienne dans ce pays et cette manifestation est une véritable révolution des mentalités. Même si déjà la 
présence féminine était importante dans toutes les luttes, c'est une étape supplémentaire. Il y avait eu les 3000 ouvrières de l'usine Mehalla al-Koubra en 2006 qui ont joué un rôle important dans l'histoire de la révolution ensuite, bien d'autres, dans d'autres grèves, et leur présence jour et nuit place Tahrir.

Il y a environ un mois, il y a eu une manifestation de femmes yéménites qui ont brûlé leur voile en protestation contre les violences qu'elles subissent. Il y a eu aussi cette jeune égyptienne qui s'était montrée nue sur son site internet en signe de protestation, ce qui a «fait le buzz » mais surtout entraîné le même geste de solidarité de vingt femmes israéliennes avec le slogan «n'ayez plus peur »!

Mais là, c'est qualitativement différent. Puisse cette manifestation redonner naissance et force au mouvement féministe égyptien (qui fut un des premiers au monde après la révolution de 1919) et arabe tout entier et, pourquoi pas, encore ailleurs !

Petit détail amusant, la manifestation est passée devant la Cour suprême où les Frères Musulmans, le Wafd et Kefaya faisaient un sit-in pour une commission d'enquête contre les violences policières en décembre. À l'approche de la manifestation, ils sont partis, pour que ça ne crée pas d'incidents devant la Cour suprême. Il faut respecter les institutions, n'est-ce pas! Les Frères musulmans et les salafistes n'ont pas participé à cette manifestation féminine. Ils étaient brocardés par certaines femmes, choisissant, comme le disaient des manifestantes, la protection de leurs résultats électoraux plutôt que l'honneur des femmes. Tout un programme.

Dans la manifestation, la tonalité n'était plus seulement à un gouvernement civil tout de suite mais à l'arrestation et au jugement des militaires. Les militaires ont complètement perdu, pour le moment en tous cas, la campagne d'intox et leur bras de fer contre les révolutionnaires. Ils se sont excusés publiquement. Après, il est vrai, qu'Hillary Clinton l'ait demandé. (Il faudrait qu'elle le demande aussi pour TF1 qui avait repris les calomnies des militaires et ne s'est pas excusé...).

111 Certains des « électoralistes » pathologiques s'accrochent à l'idée que ce n'est pas parce que les gens manifestent contre les violences policières qu'ils retirent toute légitimité à l'armée et aux élections. Certes, mais ça en retire pas mal.

112 La révolution continue et s'approfondit.

\section{NOTES}

1. «Égypte : Notes sur une révolution en marche ». J. Chastaing. Carré Rouge, n 45, www.carrerouge.org

2. Formule emblématique de la révolte des Canuts qui a tant touché Stendhal et apeuré Flaubert.

3. Les participations " pharaoniques » telles qu'elles sont présentées se réduisent dans la réalité à $48 \%$ de participation pour la Tunisie, 13 à $20 \%$ pour le Maroc, $48 \%$ environ (pour les chiffres donnés pour le moment) pour l'Egypte où le vote est pourtant obligatoire. C'est dire la légitimité toute relative de ces scrutins et les limites d'une soi-disant vague islamiste.

4. République Arabe Unie (nom porté par l'Egypte jusqu'en 1971), projet de Nasser de fonder des États-Unis socialistes arabes, commencé avec la Syrie à la fin des années 1950, un peu plus tard 
avec le Yémen, déclenchant fierté et espoir dans les peuples et des soulèvements comme au Liban, autour d'un socialisme arabe nassérien niant la lutte de classe mais associant patrons et ouvriers dans le travail productif qui aurait été facteur de justice sociale et d'égalité.

\section{AUTEUR}

\section{JACQUES CHASTAING}

Animateur de la revue Carré rouge et de son site web www.carre-rouge.org 